\title{
HYDRODYNAMICAL MODELLING OF T PYXIDIS
}

\author{
Guillermo García-Segura, ${ }_{1}^{1}$ Mordecai-Mark Mac Low, ${ }^{2}$ and Michael M. Shara ${ }^{2}$
}

The Hubble Space Telescope observations showed that some nova outbursts may not produce smooth shells of gas, but thousands of gaseous blobs. In the case of $T$ Pyx the blobs are distributed in eight concentric circles around the exploding star, producing a pattern similar to tree rings. We explore here the formation of the tree rings by hydrodynamical simulations.

The simulations have been performed using the magnetohydrodynamic code ZEUS-3D (version 3.4), developed by M. L. Norman and the Laboratory for Computational Astrophysics (Stone \& Norman 1992). We perform the simulations in spherical coordinates $(r, \theta, \phi)$ with periodic boundary conditions in $\phi$, and rotational symmetry assumed with respect to $\theta$. Thus, our models are effectively two-dimensional, and the simulations are carried out in the equatorial $(r, \phi)$ plane. The models include the Raymond \& Smith (1977) cooling curve above $10^{4} \mathrm{~K}$. For temperatures below $10^{4} \mathrm{~K}$, the shocked gas region is allowed to cool down with the radiative cooling curves given by Dalgarno \& McCray (1972) and MacDonald \& Bailey (1981).

As initial conditions for the computations, we assume that there is no background wind from the red dwarf in T Pyx, but only material from the previous nova outbursts. Our best guess as to the mass loss is that it dies away as either $1 / t$ or $1 / t^{2}$ down to extremely low values within $0.5 \mathrm{yr}$ after the explosion. The velocity also dies away, though, from 2000 down to $10 \mathrm{~km} / \mathrm{s}$ over the same time period, so the next blast finds a stretched out wind to run in to. The functional dependence of $\dot{M}$ and $v_{\infty}$ versus time it is a simple linear model:

$$
\begin{aligned}
& \dot{M}(t)=2 \times 10^{-6} \mathrm{M}_{\odot} \mathrm{yr}^{-1} \times[0.5-t] \text { for } t(y r) \leq 0.5 \\
& v_{\infty}(t)=4000 \mathrm{~km} \mathrm{~s}^{-1} \times[0.5-t] \text { fort }(y r) \leq 0.5
\end{aligned}
$$

Then a repetition each $30 \mathrm{yr}$. We did first a run, in order to get the densities and pressures of the large hot bubble. After that, we did a run (Figure 1) inside the bubble, with density of $5 \times 10^{-26} \mathrm{~g} \mathrm{~cm}^{-3}$ and energy density of $2 \times 10^{-10}$, keeping pressurized the outer boundary.

As one can notice, the clumps form in the ejecta,

\footnotetext{
${ }^{1}$ Instituto de Astronomía-UNAM.

${ }^{2}$ American Museum of Natural History.
}
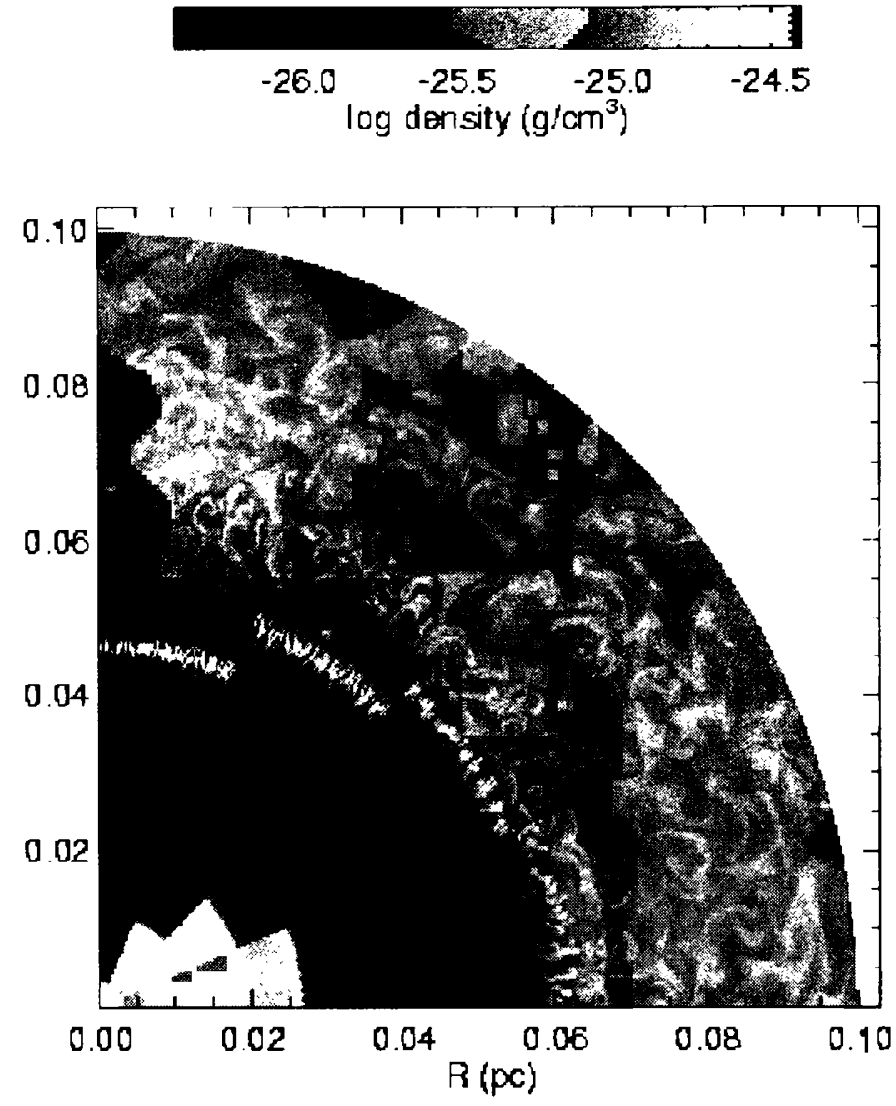

Fig. 1. Run with $800 \times 180$ zones $(r, \phi)$ (22.5 degrees). The time step in the plot is 5 years, covering 15 years. The plot shows the the collision of the 18th explosion with the reverse shock, and the first 15 years of the 19 th explosion.

they are compressed in the inner shock, and after that, they get diluted. Something interesting is the brightening (higher density, higher temperature) of the clumps at the arrival of the collision, which probably matches Shara's 1997 paper with a few years of baseline. It is very interesting how the clumps slow down at the reverse shock, because Shara et al.(1997) finds that the T Pyx blobs have proper motions under $20 \mathrm{~km} \mathrm{~s}^{-1}$.

\section{REFERENCES}

Dalgarno, A. \& McCray, R. A. 1972, ARAA, 10, 375 MacDonald, J. \& Bailey, M. E. 1981, MNRAS, 197, 995 Raymond, J. C. \& Smith, B. W. 1977, ApJS, 35, 419 Shara et al. 1997, AJ, 114, 258

Stone, J. M. \& Norman, M. L. 1992, ApJS, 80, 753 